EPJ Web of Conferences 38, 04005 (2012)

DOI: $10.1051 /$ epjconf/20123804005

(C) Owned by the authors, published by EDP Sciences, 2012

\title{
Microscopic calculations beyond mean-field with zero-range effective interactions
}

\author{
M. Brenna ${ }^{1,2, a}$, G. Colò ${ }^{1,2}$, P. F. Bortignon ${ }^{1,2}$, and X. Roca-Maza ${ }^{2}$ \\ 1 Dipartimento di Fisica, Università degli Studi di Milano, via Celoria 16, I-20133 Milano, Italy \\ 2 INFN, Sezione di Milano, via Celoria 16, I-20133 Milano, Italy
}

\begin{abstract}
A completely microscopic formalism has been developed to treat the observable properties of singleparticle states (e.g. energies and spectroscopic factors), as well as of collective states (e.g., their energy centroid, their damping width and their gamma decay to the ground state or to low lying states) beyond mean-field. The phonons are calculated within the fully self-consistent random phase approximation (RPA). The particle-vibration coupling (PVC) is accounted for within the framework of the nuclear field theory (NFT) at the lowest contributing order. All the calculations have been performed using the whole effective Skyrme interaction.
\end{abstract}

\section{Introduction}

The idea that the nucleons behave as independently moving particles in an average potential, is the basis of selfconsistent mean-field (SCMF) models. These models are in many respects analogs of the density-functional theory (DFT), which gives a very successful description of all kinds of many-electron systems. Contrary to the electronic case, in which electronic energy functionals of high accuracy may be derived ab initio from electron gas theory, nuclear SCMF models employ effective interactions which are adjusted by extensive fits to nuclear structure data. Nowadays, both in the non-relativistic and in the covariant framework, SCMF approaches uses rather sophisticated functionals. Such an approach can produce good results for bulk nuclear properties like masses, radii and deformations, covering almost the whole chart of nuclides, also in the super-heavy and super-deformed regions. A review of SCMF models can be found in Ref [1].

Moreover, the time dependent extension of stationary mean-field models, or time dependent DFT, is formally straightforward. The linearization of the time-dependent mean-field equations bring to the Random Phase Approximation (RPA) equations. Those describe the dynamics of the nucleus as a whole, as a coherent superposition of one particle-one hole excitation. In particular, the RPA is one of the most successful theories for the description of nuclear excitations in the energy range of giant resonances (GR).

Nuclear giant multipole resonances had been widely investigated in the past decades both theoretically and experimentally (see, e.g., [2,3]). They carry definite quantum numbers (spatial angular momentum $\mathrm{L}$, spin $\mathrm{S}$, isospin $\mathrm{T}$ ) and, as a rule, they exhaust a large fraction of the associated energy-weighted sum rule. Accordingly, the macroscopic picture of a giant resonance is often thought to be that of a coherent motion of all nucleons. These states have finite lifetime and consequently they carry a width of the order of 3-5 MeV. The most probable damping mechanism

\footnotetext{
a e-mail: marco.brenna@mi.infn.it
}

is their coupling to progressively more complicated states (of $2 \mathrm{p}-2 \mathrm{~h}, 3 \mathrm{p}-3 \mathrm{~h}, \ldots$.. np-nh character). The associated contribution to the total width, called spreading width $\left(\Gamma^{\downarrow}\right)$ is the dominant one. GRs lie in general above the threshold for emitting a nucleon, so they can decay directly by emission of particles, mainly neutrons in medium-heavy nuclei (being proton emission hindered by the large Coulomb barrier). The corresponding escape width $\left(\Gamma^{\uparrow}\right)$ is of some relevance in light nuclei but much less important in heavy nuclei. Eventually, the $\gamma$-decay width $\left(\Gamma_{\gamma}\right)$, given by coupling to the electromagnetic field is a small fraction $\left(\approx 10^{-3}\right)$ of the total width. Despite this, the study of the $\gamma$ decay of GRs has been considered a valuable tool for about 30 years [4,5].

Nevertheless, it is known that SCMF models need to be developed more to overcome some limitations. First, they show a lower density of states around the Fermi energy than measured. Some features, like the single particle states, the spectroscopic factors or the width of GRs, do not belong strictly to the DFT framework. Regarding this, we still lack a fully self-consistent microscopic model that is able to describe single-particle properties along the isotopic table.

To improve further on the quality of the description of the nuclear structure, one of the route that can be undertaken at this point is to introduce correlations beyond the mean field, allowing the interweaving between the dynamics of the mean-field, i.e. the phonons, and the single particle degrees of freedom. Widely used theoretical approaches are the quasi-particle-phonon model (QPM) [6], the extended theory of finite Fermi system (ETFFS) [7], and the nuclear field theory (NFT) [8,9], based on the particle-vibration coupling (PVC) idea. The NFT, that we will adopt in this paper, provides us with a consistent and perturbative framework in which phonons and single-particle degrees of freedom are considered as the relevant independent building blocks of the low-lying spectrum of finite nuclei.

We have developed a completely microscopic self-consistent model, based on Skyrme functionals, to treat properly single-particle states [10] and collective observables, 


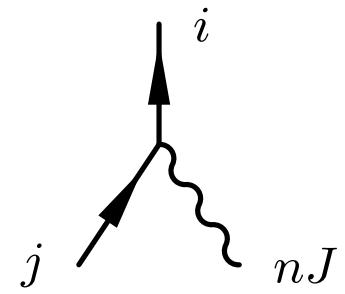

Figure 1. The basic particle-vibration vertex.

like the energy centroid of the resonances, the strength function (or the spreading width) and $\gamma$-decay width [11]. In our approach we adopt the fully self-consistent nonrelativistic mean-field (MF) approach based on Skyrme HartreeFock (HF) plus RPA [12].

In this paper, we apply this model for the study of two observables: the strength function of the isoscalar giant quadrupole resonance (ISGQR) and of the isovector giant quadrupole resonance (IVGQR) in ${ }^{208} \mathrm{~Pb}$; and the $\gamma$-decay width of the ISGQR to the ground state and the low-lying octupole state in ${ }^{208} \mathrm{~Pb}$. There has been recently a renewed interest for the latter, following the implementation of the new AGATA detector array [13].

In Sec. 2 we briefly summarize the basic features of our formalism and in Sec. 3 we give our results. Eventually, we draw our conclusions in Sec. 4.

\section{Formalism}

In this section we briefly sketch our theoretical framework (for further details, see Refs.[10,11]).

In the case of the coupling with density vibration, the basic vertex depicted in figure 1 can be calculated starting from the RPA representation of the $n$th phonon with multipolarity $L$ (here $L$ is the same as the total angular momentum $J$ and parity is the natural one). The resulting PVC vertex reads

$$
\begin{aligned}
\langle i\|V\| j, n J\rangle=\sqrt{2 J+1} & \sum_{p h} X_{p h}^{n J} V_{J}(i h j p) \\
& +(-)^{j_{h}-j_{p}+J} Y_{p h}^{n J} V_{J}(i p j h),
\end{aligned}
$$

where $X_{p h}^{n J}, Y_{p h}^{n J}$ are the forward and backward RPA amplitudes [14], and $V_{J}$ is the particle-hole coupled matrix element

$$
\begin{aligned}
V_{J}(\text { ihjp })=\sum_{\{m\}}(-)^{j_{j}-m_{j}+j_{h}-m_{h}} & \left\langle j_{i} m_{i} j_{j}-m_{j} \mid J M\right\rangle \\
& \times\left\langle j_{p} m_{p} j_{h}-m_{h} \mid J M\right\rangle \\
& \times\left\langle j_{i} m_{i} j_{h} m_{h}|V| j_{j} m_{j} j_{p} m_{p}\right\rangle .
\end{aligned}
$$

For the detailed derivation of the the reduced matrix element of Eq. (1), we refer to the Appendix of Ref. [10].

\subsection{The strength function}

It has been known for several decades that coupling with low-lying vibrations is the main source of the GR width [15,7]. In Ref. [16], calculations of the GRs strength functions that take into account this coupling were performed, based on the use of a phenomenological separable force

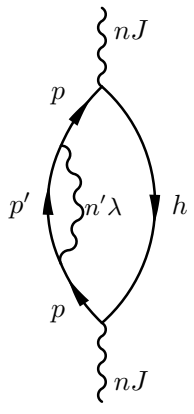

$\Sigma_{s-e}$

(a)

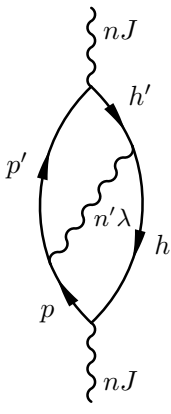

$\Sigma_{v}$

(b)
Figure 2. Processes associated with the strength of the $|n J\rangle$ RPA state. Similar diagrams are obtained by interchanging the role of particles and holes.

in the surface coupling model. We perform a similar calculation here by using consistently the Skyrme force in both $\mathrm{HF}+\mathrm{RPA}$ procedures and in the PVC vertex. More recently, a microscopic, though not self-consistent calculation were undertaken in Ref. [17].

The perturbative NFT diagrams that contribute to the strength function can be divided into two groups, and a representative of each group is depicted in figure 2 . The diagram in figure 2(a) is the self-energy of the particle (hole) that form the phonon state, i.e. it represents the process in which the particle (hole) creates and subsequently reabsorbs the intermediate vibration of multipolarity $\lambda$. Figure 2 (b) represents the process in which a $\lambda$-pole phonon is exchanged between the particle and the hole. If $|n J\rangle$ or $|n \lambda\rangle$ is a density oscillation, the latter contributions have opposite sign with respect to the former one [15].

The probability of finding the resonance state per unit energy can be written as [16]

$$
P(E)=\frac{1}{2 \pi} \frac{\Gamma_{\mathrm{GR}}+\eta}{\left(E-E_{\mathrm{GR}}-\Delta E_{G R}\right)^{2}+\left(\frac{\Gamma_{\mathrm{GR}}+\eta}{2}\right)^{2}},
$$

where $\Delta E_{\mathrm{GR}}$ is the real part of the sum of the diagrams in figure 2, while $\Gamma_{G R}$ is the imaginary part of the same sum. The parameter $\eta$ corresponds to the energy interval over which averages are taken and represents, in an approximate way, the coupling of the intermediate states to more complicated configurations. In our calculation we set this parameter at $1 \mathrm{MeV}$.

The two diagrams in figure 2(a) and 2(b) are evaluated as follows:

$$
\begin{aligned}
& \Sigma_{s-e}\left(G R, E_{J}\right)=\sum_{p p^{\prime} h n^{\prime}} \frac{1}{(2 J+1)(2 \lambda+1)} \\
& \times \frac{|\langle p\|V\| h, n J\rangle|^{2}\left|\left\langle p\|V\| p^{\prime}, n^{\prime} \lambda\right\rangle\right|^{2}}{\left(E_{J}-\epsilon_{p h}+i \eta\right)^{2}\left(E_{J}-E_{n^{\prime}}-\epsilon_{p^{\prime} h}+i \eta^{\prime}\right)}, \\
& \Sigma_{v}\left(G R, E_{J}\right)=\sum_{p p^{\prime} h h^{\prime} n^{\prime}} \frac{(-)^{j_{p}+j_{h}+j_{p^{\prime}}+j_{h^{\prime}}}}{2 J+1}\left\{\begin{array}{lll}
j_{h} & j_{p} & J \\
j_{p^{\prime}} & j_{h^{\prime}} & \lambda
\end{array}\right\} \\
& \times \frac{\langle p\|V\| h, n J\rangle\left\langle h^{\prime}, n J\|V\| p^{\prime}\right\rangle\left\langle h\|V\| h^{\prime}, n^{\prime} \lambda\right\rangle\left\langle p^{\prime}, n^{\prime} \lambda\|V\| p\right\rangle}{\left(E_{J}-\epsilon_{p h}+i \eta\right)\left(E_{J}-\epsilon_{p^{\prime} h^{\prime}}+i \eta\right)\left(E_{J}-E_{n^{\prime}}-\epsilon_{p^{\prime} h}+i \eta^{\prime}\right)} \text {. }
\end{aligned}
$$

In these equations $\epsilon_{p h}$ is equal to the difference of the Hartree-Fock (HF) single-particle energies $\epsilon_{p}-\epsilon_{h}$, and $V$ 
is the residual particle-hole interaction. The other contributions are obtained interchanging the role of particles and holes, applying the usual rules of diagrammatic expansion.

\subsection{The $\gamma$ decay}

The transition amplitude for the emission of a photon of a given multipolarity is proportional to the matrix element of the electromagnetic operator, that takes the form

$$
Q_{\lambda \mu}=\frac{1}{2} \sum_{i=1}^{A} e_{i}^{e f f} i^{\lambda} r_{i}^{\lambda} Y_{\lambda \mu}\left(\hat{\boldsymbol{r}}_{i}\right)
$$

In this equation, the effective charge $e_{i}^{\text {eff }}$ for neutrons and protons is introduced (see, e.g., Ref [18]), since the recoil of the nucleus is non negligible, in general.

The gamma decay width, summed over the magnetic substates of the photon and of the final nuclear state, is then given by

$$
\Gamma_{\gamma}(E \lambda ; i \rightarrow f)=\frac{8 \pi(\lambda+1)}{\lambda[(2 \lambda+1) ! !]^{2}}\left(\frac{E}{\hbar c}\right)^{2 \lambda+1} B(E \lambda ; i \rightarrow f),
$$

where $E$ is the energy of the transition and the reduced transition probability $B$ associated with the above operator $Q_{\lambda \mu}$ is

$$
B(E \lambda ; i \rightarrow f)=\frac{1}{2 J_{i}+1}\left|\left\langle J_{f}\left\|Q_{\lambda}\right\| J_{i}\right\rangle\right|^{2} .
$$

In this work we consider only the direct $\gamma$ decay. To allow a comparison with the experiment, also the $\gamma$ decay of the compound nucleus have to be taken into account $[2,19]$.

\subsubsection{The $\gamma$ decay to the ground state}

We consider in this section the decay of an excited RPA state (which can be, e.g., a GR) to the ground state. We limit ourselves to spherical systems. The RPA states have quantum numbers $J M$ (we consider natural parity, or nonspinflip, states) and, in addition, they are labelled by an index $n$.

At the RPA level, in the case of the decay of the state $|n J\rangle$ to the ground-state, we obtain for the reduced matrix of Eq. (7),

$$
\left\langle 0\left\|Q_{J}\right\| n J\right\rangle=\sum_{p h}\left(X_{p h}^{n J}+Y_{p h}^{n J}\right) e_{p h}^{e f f}\left\langle j_{p}\left\|i^{J} r^{J} Y_{J}\right\| j_{h}\right\rangle,
$$

where $X(Y)$ are the forward (backward) RPA amplitudes. It is possible to give a diagrammatic representation of the ground state decay (see figure 3 ).

\subsubsection{The $\gamma$ decay to low-lying state}

The decay to low-lying states is an intrinsically beyondmean-field process, as RPA is by construction an appropriate theory to describe transitions between states that differ only by one vibrational state (phonon). For other processes, like the one at hand, the extension to a treatment

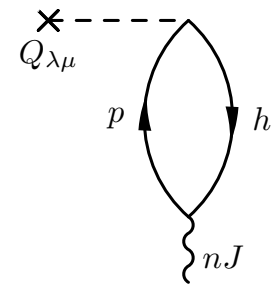

(a)

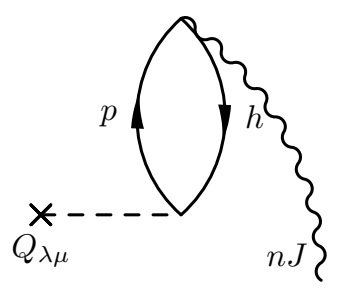

(b)
Figure 3. Diagrams representing the decay of the vibrational state $|n J\rangle$ state to the ground state.

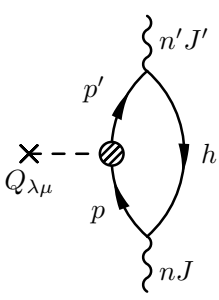

(a)

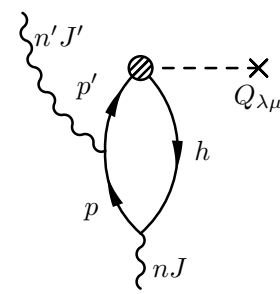

(b)

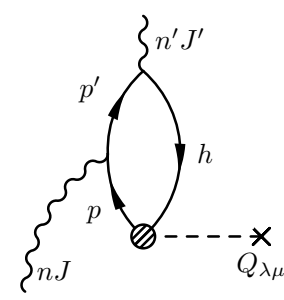

(c)
Figure 4. A set of diagrams representing the decay of the vibrational state $|n J\rangle$ state to a low-lying $\left|n^{\prime} J^{\prime}\right\rangle$ state. Other diagrams are obtained interchanging the role of particles and holes.

beyond RPA is mandatory. As stated above, the NFT provides a consistent framework in which such processes can be treated. We consider all the lowest-order contributions to the transition amplitude between two different phonon states, evaluating all the lowest-order perturbative diagrams involving single-particle states and phonon states, which can lead from the initial state to the final state by the action of the external electromagnetic field. The PVC idea has already been applied to the calculation of the $\gamma$ decay [20]; however, the main difference is that in this work we use in a consistent way a microscopic interaction of the full-Skyrme type.

The NFT diagrams associated with the $\lambda$-pole decay of the initial RPA state $|n J\rangle$ (at energy $E_{J}$ ) to the final state $\left|n^{\prime} J^{\prime}\right\rangle$ (at energy $E_{J^{\prime}}$ ) can be grouped into three families. A representative of each family is shown in figure 4 and corresponding analytical expressions are the following:

$$
\begin{gathered}
\left\langle n^{\prime} J^{\prime}\left\|Q_{\lambda}\right\| n J\right\rangle_{(\mathrm{a})}=\sum_{p p^{\prime} h}(-)^{J+\lambda+J^{\prime}+1}\left\{\begin{array}{ccc}
J & \lambda & J^{\prime} \\
j_{p^{\prime}} & j_{h} & j_{p}
\end{array}\right\} \\
\times \frac{\langle p\|V\| h, n J\rangle\left\langle h, n^{\prime} J^{\prime}\|V\| p^{\prime}\right\rangle Q_{p^{\prime} p}^{\lambda p o l}}{\left(E_{J}-\epsilon_{p h}+i \eta\right)\left(E_{J^{\prime}}-\epsilon_{p^{\prime} h}\right)}, \\
\left\langle n^{\prime} J^{\prime}\left\|Q_{\lambda}\right\| n J\right\rangle_{(\mathrm{b})}=\sum_{p p^{\prime} h}(-)\left\{\begin{array}{ccc}
J & \lambda & J^{\prime} \\
j_{p^{\prime}} & j_{p} & j_{h}
\end{array}\right\} \\
\times \frac{\langle p\|V\| h, n J\rangle\left\langle p^{\prime}, n^{\prime} J^{\prime}\|V\| p\right\rangle Q_{h p^{\prime}}^{\lambda p o l}}{\left(E_{J}-\epsilon_{p h}+i \eta\right)\left(E_{J}-E_{J^{\prime}}-\epsilon_{p^{\prime} h}+i \eta^{\prime}\right)} \\
\left\langle n^{\prime} J^{\prime}\left\|Q_{\lambda}\right\| n J\right\rangle_{(\mathrm{c})}=\sum_{p p^{\prime} h}\left\{\begin{array}{lll}
J & \lambda & J^{\prime} \\
j_{h} & j_{p^{\prime}} & j_{p}
\end{array}\right\} \\
\times \frac{\left\langle p^{\prime}\|V\| p, n J\right\rangle\left\langle h, n^{\prime} J^{\prime}\|V\| p^{\prime}\right\rangle Q_{p h}^{\lambda p o l}}{\left(E_{J^{\prime}}-\epsilon_{p^{\prime} h}\right)\left(E_{J}+\epsilon_{p h}-E_{J^{\prime}}+i \eta\right)} .
\end{gathered}
$$




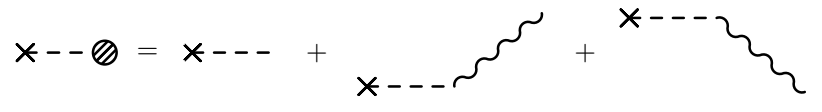

Figure 5. Polarization contribution to the operator $Q_{\lambda \mu}$.

The notation here is the same as in Sec. 2.1. The other contributions are obtained interchanging the role of particles and holes, applying the usual rules of diagrammatic expansion.

In all the above equations, the matrix elements of the operator $Q_{\lambda}$ include the contribution from the nuclear polarization (consequently they carry the label pol). In a diagrammatic way, the bare and the polarization contributions of Eq. (10) are drawn in figure 5.

$$
\begin{aligned}
Q_{i j}^{\lambda p o l}= & \left\langle i\left\|Q_{\lambda}\right\| j\right\rangle+ \\
& +\sum_{n^{\prime}} \frac{1}{\sqrt{2 \lambda+1}}\left[\frac{\left\langle 0\left\|Q_{\lambda}\right\| n^{\prime} \lambda\right\rangle\left\langle i, n^{\prime} \lambda\|V\| j\right\rangle}{\left(E_{J}-E_{J^{\prime}}\right)-E_{n^{\prime}}+i \eta}\right. \\
& \left.-\frac{\left\langle i\|V\| j, n^{\prime} \lambda\right\rangle\left\langle n^{\prime} \lambda\left\|Q_{\lambda}\right\| 0\right\rangle}{\left(E_{J}-E_{J^{\prime}}\right)+E_{n^{\prime}}+i \eta}\right],
\end{aligned}
$$

where $\left|n^{\prime} \lambda\right\rangle$ are the RPA states having multipolarity $\lambda$ (and lying at energy $E_{n^{\prime}}$ ), while the bare operator $Q_{\lambda}$ has been defined in Eq. (5). The polarization contribution, that is, the second and third term in the latter equation, has the effect of screening partially the external field.

\section{Results}

In this section, the results obtained from our numerical calculation in ${ }^{208} \mathrm{~Pb}$ are discussed. In all cases, we start by solving the HF equations in a radial mesh that extends up to $20 \mathrm{fm}$, with a radial step of $0.1 \mathrm{fm}$. Once the HF solution is found, the RPA equations are solved in the usual matrix formulation. Vibrations (or phonons) with a given multipolarity $L$ (see the following sections for more details), and with natural parity, are calculated. The isoscalar dipole state is subtracted using the procedure explained in the Appendix A of Ref. [21]. A lower cutoff on the collectivity of the intermediate phonon states is needed for at least two reasons: firstly, RPA is known to be not reliable for non-collective states, and secondly, introducing poorly collective phonons would oblige to account for the issue of the Pauli principle correction. The RPA model space must be large, due to the zero-range character of the Skyrme forces. It consists of all the occupied states, and all the unoccupied states lying below a cutoff energy $E_{C}$ equal to 50 $\mathrm{MeV}$. The states at positive energy are obtained by setting the system in a box, that is, the continuum is discretized. These states have increasing values of the radial quantum number $n$, and are calculated for those values of $l$ and $j$ that are allowed by selection rules. With this choice of the model space the energy-weighted sum rules (EWSRs) satisfy the double commutator values at the level of about $99 \%$; moreover, the energy and the fraction of EWSR of the states which are relevant for the following discussion are well converged. The same model space is used in the PVC.

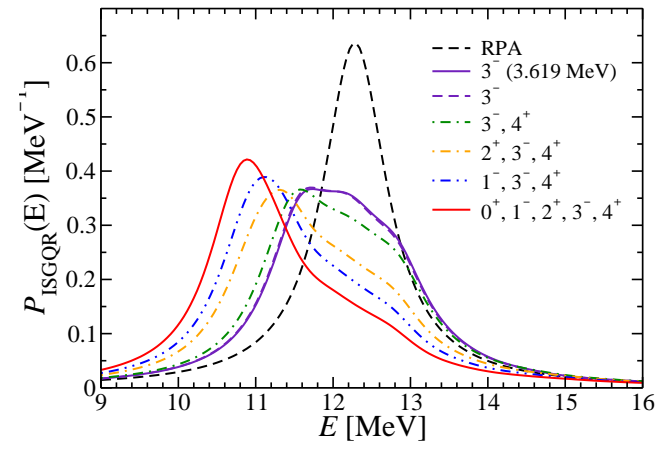

Figure 6. Probability $P$ to find the ISGQR at an energy $E$ in ${ }^{208} \mathrm{~Pb}$. Each line corresponds to the probability obtained when the phonons listed in the legend are used as intermediate states. The label RPA (black-dashed line) refers to the RPA result, in which none of the diagrams in figure 2 are taken into account, but a lorentzian averaging with functions having $1 \mathrm{MeV}$ width is introduced.

\subsection{The ISGQR and IVGQR strength functions}

In this section, the strength function for the isoscalar and isovector giant quadrupole resonances in ${ }^{208} \mathrm{~Pb}$ are discussed. The interaction used is the SLy5 [22] parametrization of a Skyrme force.

In figure 6 the probability of finding the ISGQR state, calculated by including in the diagrams an increasing number of intermediate phonon states, is displayed. In particular, phonons with multipolarity ranging from 0 to 4 , and with natural parity, are introduced. Only those with energy smaller than $30 \mathrm{MeV}$ and a fraction of energy weighted sum rule larger than $5 \%$ where selected as intermediate states. The most important contribution to the spreading width comes from the first $3^{-}$state, in agreement width previous calculations [16]. Finally, we get an energy centroid of $E=10.9 \mathrm{MeV}$ and a width $\Gamma^{\downarrow} \approx 2 \mathrm{MeV}$, in good agreement width the experimental findings [23]. A more detailed discussion can be found in [11].

The probability of finding the IVGQR state, calculated in a similar way, is shown in figure 7 . In this case, only phonons with multipolarity from 0 to 3 are included, using the same exclusion criteria. It is not possible to identify a multipolarity that affect the width more than the others. The single RPA state is splitted into two parts: while the higher energy peak is not really affected by the introduction of an increasing number of intermediate phonons, the lower energy one, conversely, becomes broader and it is shifted to lower energy. For this reason it is difficult to single out a value for the energy centroid, that we can set at 21.2 MeV, while the spreading width is of the order of 3.8 $\mathrm{MeV}$. Also these values are consistent with the more recent experimental results [24].

\subsection{The $\gamma$ decay}

In this section, the results obtained for $\gamma$ decay the ISGQR in ${ }^{208} \mathrm{~Pb}$ to the ground state and the low-lying octupole state are discussed. We use four different Skyrme interactions: SLy5 [22], SGII [25], SkP [26], LNS [27].

We group in Table 1 the main properties of the collective states involved in the processes at hand. 


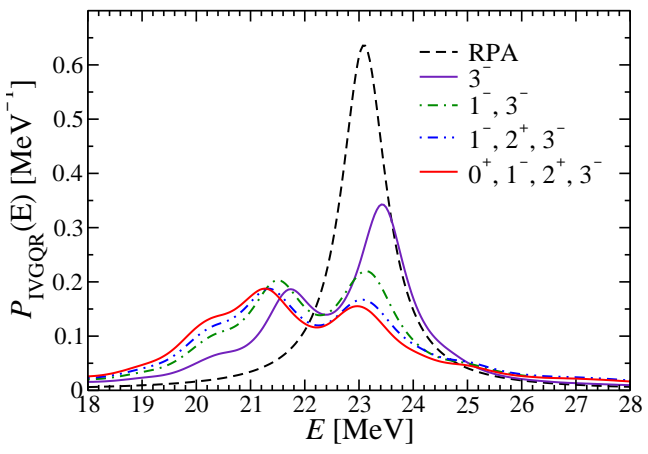

Figure 7. Probability $P$ to find the IVGQR at an energy $E$ in ${ }^{208} \mathrm{~Pb}$. Each line corresponds to the probability obtained when the phonons listed in the legend are used as intermediate states. The label RPA (black-dashed line) refers to the RPA result, in which none of the diagrams in figure 2 are taken into account, but a lorentzian averaging with functions having $1 \mathrm{MeV}$ width is introduced.

Table 1. Energy and collectivity of the ISGQR and of the lowlying $3^{-}$state in ${ }^{208} \mathrm{~Pb}$. The label Exp. indicates the corresponding experimental values (the italic number after the value is the experimental error on the last figure).

\begin{tabular}{|c|c|c|c|c|}
\hline & \multicolumn{2}{|c|}{$2^{+}$} & \multicolumn{2}{|r|}{$3^{-}$} \\
\hline & $\mathrm{E}[\mathrm{MeV}]$ & EWSR [\%] & $\mathrm{E}[\mathrm{MeV}]$ & $\mathrm{B}(\mathrm{E} 3 \uparrow)\left[\mathrm{e}^{2} \mathrm{fm}^{6}\right]$ \\
\hline Exp. & 10.93 & 100 & 2.61453 & $6.1110^{5} 9$ \\
\hline SLy5 & 12.28 & 69.27 & 3.62 & $6.5410^{5}$ \\
\hline SGII & 11.72 & 72.31 & 3.14 & $6.5810^{5}$ \\
\hline SkP & 10.28 & 81.79 & 3.29 & $5.1110^{5}$ \\
\hline LNS & 12.10 & 66.98 & 3.19 & $5.6710^{5}$ \\
\hline
\end{tabular}

\subsubsection{The $\gamma$ decay to the ground state}

The results obtained for the decay of the ISGQR to the ground state are collected in Table 2. Together with our results, we list also the experimental data [4] and previous experimental results found in the literature, using different models.

In general, our calculations reproduce the experiment quite well, without any parameter adjustment. They tend to overestimate the decay width, and this is true in particular for SLy5; however, even in this worst case, our result are still compatible with the experimental value.

This discrepancy is found to be basically due to the fact that the energy of the giant resonance do not fit accurately the experimental findings. This is because in Eq. (6) the energy of the transition is raised to the fifth power: consequently, an increase of the energy by $1 \mathrm{MeV}$ produces an increase of the gamma decay width by about $50 \%$ (at 10 $\mathrm{MeV}$ ). To substantiate this point, in the last column of Table 2 we report the values obtained for the decay width after having rescaled the ISGQR energy to the experimental value (shown in Table 1). For this reason we conclude that this kind of measurement is not able to discriminate between models more than other inclusive observables (e.g. energy).

\subsubsection{The $\gamma$ decay to low-lying $3^{-}$state}

In Table 3, the results obtained for the decay of the ISGQR to the low-lying octupole state are shown, together
Table 2. Energy $E$ of the ISGQR and $\gamma$-decay width associated with its transition to the ground-state. The first four rows correspond to the present RPA calculations. In this case, for ${ }^{208} \mathrm{~Pb}$ we show both the bare $\Gamma_{\gamma}$ from Eq. (6) as well as the renormalized value which is discussed in the main text. The next three rows report the results of previous theoretical calculations $[20,28,19]$. In the last row the experimental value from Ref. [4] is displayed, corresponding to the direct decay.

\begin{tabular}{cccc}
\hline & $E[\mathrm{MeV}]$ & $\Gamma_{\gamma}[\mathrm{eV}]$ & $\Gamma_{\gamma}^{r e n}[\mathrm{eV}]$ \\
\hline SLy5 & 12.28 & 231.54 & 127.58 \\
SGII & 11.72 & 163.22 & 113.57 \\
SkP & 10.28 & 119.18 & 159.72 \\
LNS & 12.10 & 176.57 & 104.74 \\
\hline Ref. [19] & 11.20 & \multicolumn{2}{c}{175} \\
Ref. [20] & 11.20 & 142 \\
Ref. [28] & 10.60 & \multicolumn{2}{c}{112} \\
\hline Ref. [4] & 10.60 & \multicolumn{2}{c}{$130 \pm 40$} \\
\hline
\end{tabular}

Table 3. Decay width to the low-lying $3^{-}$for the interactions used, calculated including beyond RPA contributions. The results from Ref. [28] and Ref. [20] are also listed and in the last row, and the experimental value from Ref. [4] is provided as well.

\begin{tabular}{ccc}
\hline & $E_{\text {trans }}[\mathrm{MeV}]$ & $\Gamma_{\gamma}[\mathrm{eV}]$ \\
\hline SLy5 & 8.66 & 3.39 \\
SGII & 8.58 & 29.18 \\
SkP & 6.99 & 8.34 \\
LNS & 8.90 & 39.87 \\
\hline Ref. [20] & 8.59 & 3.5 \\
Ref. [28] & 7.99 & 4 \\
\hline Ref. [4] & 7.99 & $5 \pm 5$ \\
\hline
\end{tabular}

with the experimental result [4] and theoretical values from Refs. [20,28]. These latter are obtained using different models: in [28], the ETFFS with a separable interaction is used, while in [20], the nuclear field theory with a separable interaction at the particle-vibration coupling vertex is implemented.

Only two interactions, namely SLy5 and SkP are quantitatively consistent with the experimental findings, even though all the models are qualitatively consistent with them, since all the interaction produce a total $\Gamma_{\gamma}\left(\mathrm{GQR} \rightarrow 3^{-}\right)$ which is only a few percent of the corresponding $\Gamma_{\gamma}(\mathrm{GQR} \rightarrow$ g.s. $)$.

The most important effect that brings the $\gamma$-decay width to few electronvolts is the polarization of the nuclear medium, expressed in Eq. (10). In particular, in the case at hand, the intermediate states are dipole phonons, especially the isovector dipole resonance. The effect of this term is to screen partially the external electromagnetic field, producing a quenching factor of the order of 4 to 10, depending on the interaction used. We address the interested reader to Ref. [11] for a more detailed analysis of these results.

\section{Conclusions}

In this work we deal with some applications of our new microscopic and consistent framework based on Skyrme 
functionals. In particular, we focus our attention on the strength function of giant resonances and the $\gamma$ decay of GRs to the ground state and to low-lying collective states. These topics were studied in the past decades using phenomenological or not completely consistent models. Therefore, we implemented a scheme in which the single particle states are obtained within HF, the vibrations are calculated using fully self-consistent RPA and the whole Skyrme force is employed at the particle-vibration vertices. The decay width to the ground state is treated within the fully selfconsistent RPA, while the strength function and the decay to low-lying collective vibrations at the lowest contributing order of perturbation theory beyond RPA.

We applied our model to calculate the strength function of the isoscalar and isovector giant quadrupole resonance and to the $\gamma$ decay of the ISGQR to the ground state and the first low-lying collective octupole state in ${ }^{208} \mathrm{~Pb}$.

The energy centroid and the spreading width are consistent with the experimental findings for both the resonances. In the case of the ISGQR, the most important contribution is given by the first $3^{-}$state as intermediate state, as we expected from previous studies based on a phenomenological model. Concerning the IVGQR, the introduction of intermediate states produces a splitting of the strength function into two different peaks. Only the lower energy peak is affected introducing more and more phonons, even if we cannot identify a multipolarity that gives a major contribution.

In the $\gamma$ decay to the ground state, we found that all the interactions used give a decay width of the order of hundreds of electronvolts, in agreement with the experimental result. The small discrepancies are due to the fact that the energy of the resonance is overestimated and the decay width is highly energy dependent. Scaling the results to the experimental energy, the decay width turns out to be completely consistent with the experiment. Thus, the decay to the ground state is not able to discriminate between different models more than less inclusive observables, like the energy.

However, the $\gamma$-decay to low-lying collective states is more sensitive to the interaction used. As a matter of fact, only two interactions (namely SLy5 and SkP) manage to achieve a decay width of few electronvolts, consistently with the experimental findings. Nonetheless, the other interactions give a width $\Gamma_{\gamma}$ that is of the order of tens of electronvolts. It is quite remarkable that our calculation, being parameter-free, reproduces numbers that are several orders of magnitude smaller than the nuclear scale of $\approx$ $\mathrm{MeV}$. In particular, the description of the dipole spectrum is a crucial point, because small differences in the strength of the dipole states, introduced as intermediate states, change significantly the polarization of the nuclear medium.

The support of the Italian Research Project "Manybody theory of nuclear systems and implications on the physics of neutron stars" (PRIN 2008) is acknowledged.

\section{References}

1. M. Bender, P. H. Heenen, P. G. Reinhard, Rev. Mod. Phys. 75, 121 (2003)

2. P. F. Bortignon, A. Bracco, R. A. Broglia, Giant Resonances: Nuclear structure at finite temperature, Con- temporary Concepts in Physics (Harwood Academic publisher, 1998)

3. M. N. Harakeh, A. van der Woude, Giant Resonances: fundamental high-frequency modes of nuclear excitation, Oxford Studies in Nuclear Physics (Oxford University Press Inc., 2001)

4. J. R. Beene, F. E. Bertrand, M. L. Halbert, R. L. Auble, D. C. Hensley, D. J. Hören, R. L. Robinson, R. O. Sayer, T. P. Sjoreen, Phys. Rev. C 39, 1307 (1989)

5. J. R. Beene, F. E. Bertrand, D. J. Hören, R. L. Auble, B. Burks, J. Gomez del Campo, M. L. Halbert, R. O. Sayer, W. Mittig, Y. Schutz et al., Phys. Rev. C 41, 920 (1990)

6. V. G. Soloviev, Theory of Atomic Nuclei: Quasiparticles and Phonons (Institute of Physics, 1992)

7. S. Kamerdzhiev, J. Speth, G. Tertychny, Phys. Rep. 393, 1 (2004)

8. R. A. Broglia, B. R. Mottelson, D. R. Bes, R. Liotta, H. M. Sofia, Phys. Lett. B 64, 29 (1976)

9. P. F. Bortignon, R. A. Broglia, D. R. Bes, R. Liotta, Phys. Rep. 30, 305 (1977)

10. G. Colò, H. Sagawa, P. F. Bortignon, Phys. Rev. C 82, $1(2010)$

11. M. Brenna, G. Colò, P. F. Bortignon, Phys. Rev. C 85, 014305 (2012)

12. G. Colò, L. G. Cao, N. Van Giai, L. Capelli, Comput. Phys. Comm. (2012), doi: 10.1016/j.cpc.2012.07.016

13. R. Nicolini, A. Bracco, D. Mengoni, S. Leoni, F. Camera, D. Bazzacco, E. Farnea, A. Gadea, F. Birocchi, A. Camplani et al., Acta Phys. Pol. B 42, 653 (2011)

14. D. J. Rowe, Nuclear Collective Motion (Methuen and Co. Ltd., 1970)

15. G. F. Bertsch, P. F. Bortignon, R. A. Broglia, Rev. Mod. Phys. 55, 287 (1983)

16. P. F. Bortignon, R. A. Broglia, Nucl. Phys. A 371, 405 (1981)

17. G. Colò, N. Van Giai, P. F. Bortignon, Phys. Rev. C 50, 1496 (1994)

18. A. de Shalit, H. Feshbach, Theoretical Nuclear Physics: Nuclear Structure, Vol. 1 (John Wiley and Sons Inc., 1974)

19. J. R. Beene, G. F. Bertsch, P. F. Bortignon, R. A. Broglia, Phys. Lett. B 164, 19 (1985)

20. P. F. Bortignon, R. A. Broglia, G. F. Bertsch, Phys. Lett. B 148, 20 (1984)

21. X. Roca-Maza, G. Pozzi, M. Brenna, K. Mizuyama, G. Colò, Phys. Rev. C 85, 1 (2012)

22. E. Chabanat, P. Bonche, P. Haensel, J. Meyer, R. Schaeffer, Nucl. Phys. A 635, 231 (1998)

23. D. H. Youngblood, Y. W. Lui, H. L. Clark, B. John, Y. Tokimoto, X. Chen, Phys. Rev. C 69, 1 (2004)

24. D. Dale, R. Laszewski, R. Alarcon, Phys. Rev. Lett. 68, 3507 (1992)

25. N. Van Giai, H. Sagawa, Nucl. Phys. A 371, 1 (1981)

26. J. Dobaczewski, H. Flocard, J. Treiner, Nucl. Phys. A 422, 103 (1984)

27. L. G. Cao, U. Lombardo, C. Shen, N. Van Giai, Phys. Rev. C 73, 1 (2006)

28. J. Speth, D. Cha, V. Klemt, J. Wambach, Phys. Rev. C 31, 2310 (1985) 